\section{ANDES

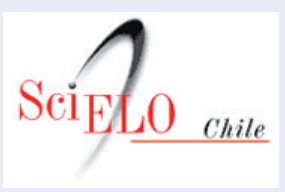

www.scielo.cl

\title{
Alteraciones de la columna vertebral en el tétanos del niño mayor. Publicado en Revista Chilena de Pediatría 1955
}

\author{
Vertebral involvement in older child's tetanus. \\ Published in en Revista Chilena de Pediatría 1955
}

\author{
Autores: Julio Hasbun S. y Agustín Muñoz G. \\ Catedra de Pediatría del Prof. Julio Meneghello R. Servicio de Radiología. Hospital Manuel Arriarán.
}

\author{
Comentario: Aníbal Espinoza Ga ${ }^{\text {a,b }}$, Luisa Schonhaut B. ${ }^{\circledR a}$ \\ aClínica Alemana, Facultad de Medicina Universidad del Desarrollo, Santiago, Chile \\ bDepartamento de Radiología, Hospital San Borja-Arriarán
}

\section{Introducción}

El tétanos es una enfermedad toxinfecciosa conocida desde la antigüedad. A pesar de que actualmente existen medidas efectivas de profilaxis de control del Clostridium tetani, sigue siendo una importante causa de morbilidad, especialmente en países en desarrollo.

Se describen 3 formas de presentación: generalizada, el localizada y cefálica. El tétanos generalizado se manifiesta como contracturas musculares espasmódicas que provocan la risa sardónica por rigidez de la musculatura de la cara y el cuello y el epistótonos por contractura de los músculos abdominales, lumbares, de cadera y muslos. Una variante es el tétanos neonatal, que suele presentarse una semana después del nacimiento, siendo la puerta de entrada el cordón umbilical.

Hasta la primera mitad del siglo XX el tétanos era endémico en Chile, no obstante, no se conocía su real impacto y poco se publicaba sobre la enfermedad. Se estimaba que las tasas de incidencia y mortalidad eran inferiores a los países vecinos, debido "al progresivo mejoramiento del nivel socioeconómico, a las característi- cas de un clima templado y seco, ya que los países de más alta incidencia tienen climas tropicales o subtropicales ${ }^{1 "}$.

En una reunión de la Societé Scientifique du Chili en 1894, Federico Puga Borne manifestó que "en más de 8.000 niños enfermos atendidos en los últimos cuatro años en la Dispensaría del Hospital de San Juan de Dios, no se había presentado ningún caso de tétanos neonatal hasta los dos que pasa a reseñar ahora"2. En una casuística de 10 años, de 1945 a 1954, Garcés H y Muñoz R. reunieron 13 casos, mientras que Manterola A. y Meneghello J. describieron 25 casos entre 1944-49, "hecho que se explica porque en esa época el Hospital "Manuel Arriaran" era el único Hospital de Niños que contaba con Posta de Primeros Auxilios, a la que consultaban casi todos los casos urgentes de la ciudad de Santiago" ${ }^{3,4}$. En ese periodo, Arriagada, P., \& Pineda, O. publicaron 6 casos de tétanos generalizado atendidos en el Servicio de Pediatría del Hospital Regional de concepción ${ }^{5}$.

En los años 60 se describió un aumento de la incidencia de tétanos, con una tasa de 0,8 casos por cada 100.000 habitantes; el mayor número de registros fue en 1963 con 73 casos $^{6}$. Estos alarmantes números fueron atribuidos "1) al incremento enorme de la población

Correspondencia:

Luisa Schonhaut B.

Ischonhaut@alemana.cl 
de Santiago. 2) a la ausencia de un plan efectivo de vacunación. 3) a la falta de consulta médica oportuna debido a la crisis de medios asistenciales"7. Pero quizás este pico de incidencia se asoció también a que, en ese año el tétanos comenzó a ser de notificación obligatoria.

Ese era el contexto epidemiológico en Chile cuando Julio Hasbun S. y Agustín Muñoz G. publicaron en Revista Chilena de Pediatría de 1955 "7 casos de alteraciones post-tetánicas de la columna vertebral en niños mayores, destacando la alta frecuencia con que ellas se presentan".

El Dr. Julio Hasbun S., fue jefe de Servicio de Radiología del Hospital Arriaran, hoy Hospital Clínico San Borja Arriaran, no solo radiólogo, simultáneamente era un gran clínico, como lo recordara el Dr. Luis Cueto Sierra "siempre recuerdo el impacto en mis inicios cuando yo sospechando una neumonía y con la placa de tórax me sugirió sonriendo una glomerulonefritis, y eso fue"s. Realizó numerosas publicaciones de diversos temas en Revista Chilena de Pediatría, con un enfoque clínico-radiológico. En 1962, al trasladarse el Dr. Julio Meneghello al flamante Hospital Roberto del Rio y asumir como jefe de servicio, llevó consigo un selecto grupo de médicos, incluyendo al Dr. Hasbun.

En febrero de 2021 un gran incendio destruyo una parte importante del Hospital San Borja Arriarán, lo que obligó a una evacuación masiva, afortunadamente sin desgracias personales. La unidad de diagnóstico por imágenes, ubicada un piso bajo los dos pisos más afectados por el incendio, se vio gravemente afectada por el necesario trabajo de bomberos, los pasillos y salas de la unidad se llenaron de agua, escombros y unas cajas con radiografías viejas no parecían más que basura, en un momento en que urgía limpiar y volver a ordenar lo esencial.

Estas cajas contenían la Colección Radiológica Patrimonial del Hospital San Borja Arriarán, la que actualmente se encuentra en proceso de digitalización. Aunque no todo sobrevivió al agua del incendio, un cuerpo importante e interesante de imágenes se salvó.

Desde esta colección patrimonial, que comenzó el Dr. Juilo Hasbun, con radiografías que datan de 1935, rescatamos las radiografías originales y de buena calidad de los mismos pacientes presentados en sus manuscritos publicados en Revista Chilena de Pediatría.

A continuación, reproducimos el manuscrito "Alteraciones de la columna vertebral en el tétanos del niño mayor" publicado en 1955, editado con las imágenes originales, además de las notas clínicas del Dr. Hasbun, que, gracias a su impecable caligrafía, permitieron complementar la publicación originali.

i Hasbún J, Muñoz A. Alteraciones de la columna vertebral en el tétanos del niño mayor. Rev Chil Pediatr 1955;26(5):205-8. https://scielo.conicyt.cl/pdf/rcp/v26n5/art03.pdf

\section{Manuscrito Julio Hasbun S. y Agustín Muñoz G.}

Las lesiones producidas por el tétanos en la columna vertebral son conocidas desde principios de siglo, cuando Lehndorff llamó la atención sobre ellas. A pesar del tiempo transcurrido y la no escasa frecuencia del tétanos en diversas regiones, son pocas las publicaciones en la literatura mundial y casi todas se refieren a casos aislados. Solamente Dietrich y colaboradores, al hacer una revisión de los casos de tétanos en niños mayores durante un lapso de 18 años, logran reunir 9 de ellos con lesiones vertebrales (69\% de los sobrevivientes). Estas lesiones consisten en aplastamiento de uno o más cuerpos vertebrales dorsales, especialmente $5^{\circ}$ y $6^{\circ}$, con características que serán señaladas más adelante.

\section{Nuestra experiencia}

Entre 1948 y 1954, se hospitalizaron en el Servicio de Infecciosos del Hospital M. Arriaran, 16 niños mayores con diagnóstico de tétanos. Dos fallecieron. De los 14 restantes, a 7 se les practicó radiografía de la columna, encontrándose en todos, alteraciones que fueron desde la discreta disminución en la altura de algunos cuerpos vertebrales, hasta el aplastamiento franco.

Caso 2: J. G. V. Obs.87.340. Nino de 12 años que sufre una caída, produciéndose numerosas heridas y erosiones en la pierna izquierda. A los 11 días presenta fiebre, dolor dorsal y luego contracturas espasmódicas de la columna dorsal y gran excitación. A su ingreso se comprueba: enfermo grave, en posición pasiva, quejumbroso, febril $\left(38^{\circ}\right)$. pálido, con opistótonos de mediana intensidad y trisrmo intenso. Se hace diagnostico de tétanos y se indica suero antitetánico 40.000 $\mathrm{U}$, en una inyección intramuscular, penicilina procaína 500.000 U. cada 8 horas, la que posteriormente se cambió por cloromicetina 500 mgrs. cada 6 horas por vía rectal, a fin de evitar el estímulo que significaban las inyecciones. Como sedantes se indicaron luminal y enemas de bromuro y cloral. Aislamiento.

La evolución fue la de un cuadro grave, con gran contractura muscular permanente y crisis contracturales frecuentes que desaparecieron a los 20 días de hospitalización. El día 28 se toma radiografía de columna que muestra aplastamiento de las vertebras dorsales $4 \mathrm{a}$ 8. Al examen de la columna se aprecia cifosis dorsal aumentada. Se coloca corset de yeso durante dos meses, al cabo de los cuales no se observan modificaciones en la cifosis ni en las alteraciones radiológicas.

\section{Comentario}

La casuística presentada no es suficientemente numerosa como para obtener conclusiones; sin embargo, es llamativo el hecho que todos los niños radiografiados mostraran lesiones de la columna (50\% de los so- 


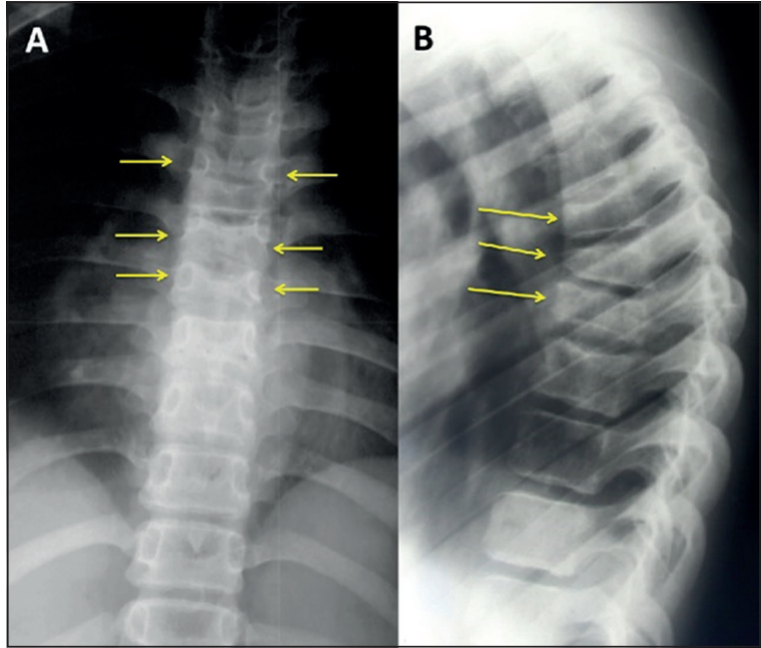

Radiografias anteroposterior (A) y lateral (B) de caso 2. Muestra fracturas vertebrales dorsales por aplastamiento cuneiformes.

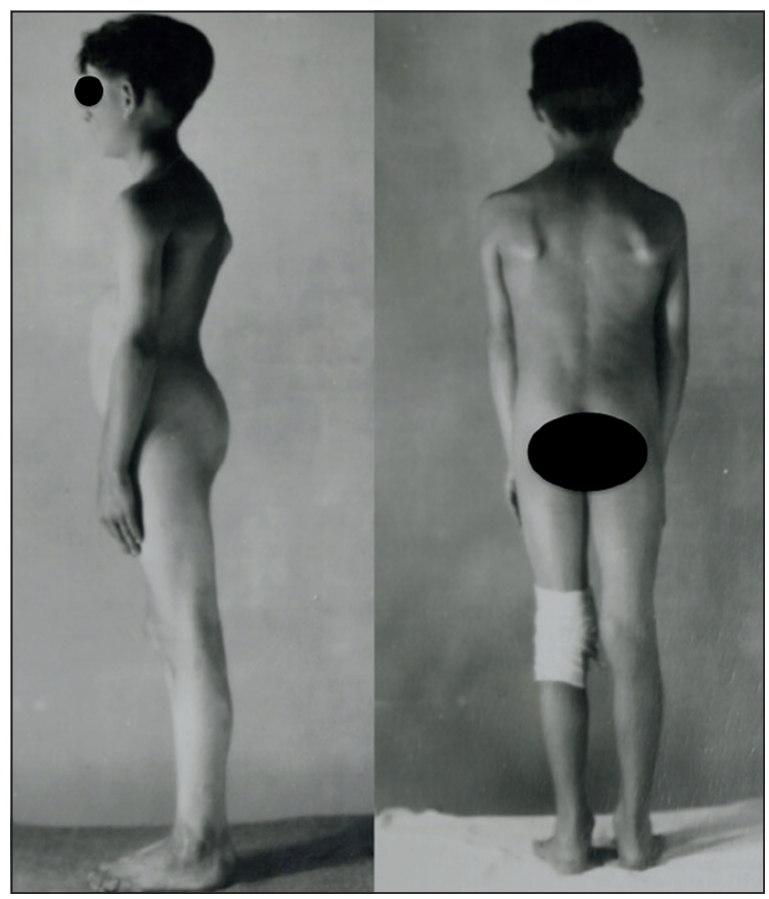

Foto de caso 2, del año 1950, en la proyección lateral es visible acentuacion de la cifosis dorsal.

brevivientes). No es difícil pensar que si hubiera sido posible obtener radiografías de todos los enfermos, el porcentaje se habría elevado sensiblemente. Creemos que las alteraciones post-tetánicas de la columna son mucho más frecuentes y pueden comprender prácticamente la totalidad de los casos de tétanos, aun los de evolución benigna, con escasa contractura muscular.

Las vertebras afectadas fueron siempre las mismas, entre D4 y D7, con mayor o exclusivo compromiso de D5 y D6. Los cuerpos vertebrales lesionados presen-

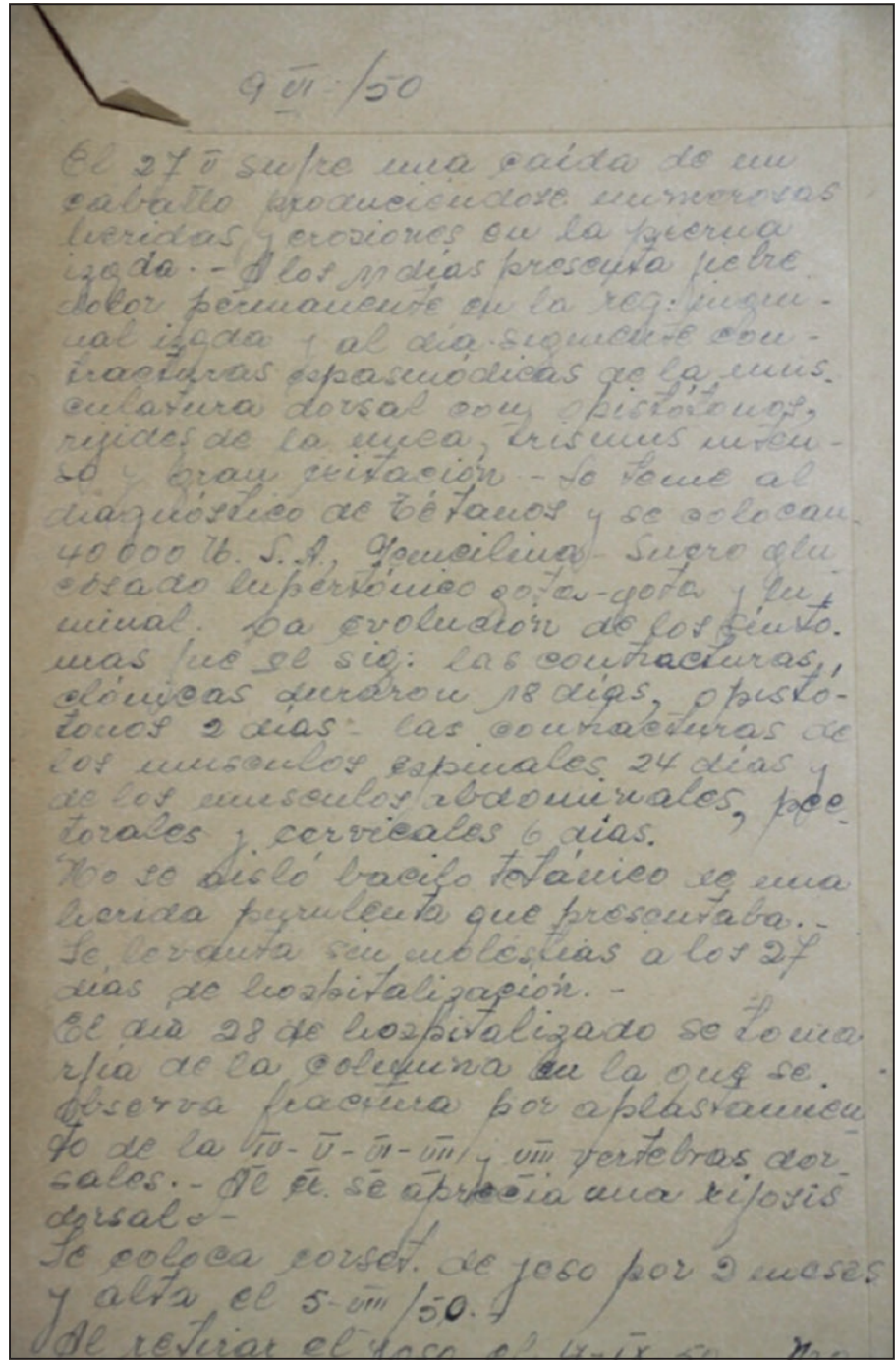

Fragmento escrito por Dr. Julio Hasbun, donde detalla los hallazgos clínicos y radiológicos del caso 2 .

taban en algunos casos la típica forma en cuña que se observa en los aplastamientos, a veces con aumento de la densidad ósea. En ninguna radiografía se observó alteración de los espacios intervertebrales ni de los pedículos. En los casos con controles posteriores hasta de 2 años, no se observó gran mejoría del aspecto radiológico.

Desde el punto de vista clínico, prácticamente no existieron manifestaciones subjetivas, y las objetivas solo se observaron en algunos niños, en forma de aumento de la cifosis dorsal o cifosis localizada, generalmente buscada en forma intencional.

Para explicar la producción de estas alteraciones se han propuesto varias teorías : 1) Teoría tóxica, según la cual. la toxina tetánica tendría acción directa sobre las vertebras produciendo osteoporosis y secundariamente una disminución de la resistencia ósea. 2) Teoría vas- 
cular: los espasmos musculares repetidos comprimirían los vasos sanguíneos perivertebrales produciendo congestión según unos, anemia según otros, condiciones que favorecerían la lesión ósea. 3) Teoría mecánica: es la más aceptable. Según ella, actúa sobre la columna una fuerza de compresión longitudinal resultante de la contracción simultánea de músculos flexores y extensores, fuerza que hace aumentar las curvaturas normales. La región dorsal queda fuertemente flectada y llegado a un limite de resistencia, los cuerpos vertebrales más débiles (los dorsales altos y medios) se aplastan. En igual forma se explican las fracturas vertebrales producidas en los diferentes tipos de convulso- terapia y en el curso de los ataques de epilepsia.

El diagnóstico diferencial no ofrece dificultades por cuanto el tétanos es una enfermedad de sintomatología muy característica, de manera que siempre se tendrá el antecedente etiológico en forma precisa.

\section{Comentario al manuscrito}

El tétatenos produjo importantes estragos en la salud de la población hasta la primera mitad del siglo XX. Si bien se contaba con la posibilidad de tratamiento con suero antitoxoide tetánico y antibióticos, su mortalidad era elevada, alcanzando cerca del $80 \%$ de los afectados'. De los sobrevivientes, la mitad sufría de complicaciones como las fracturas vertebrales, que curiosamente, provocaban poco dolor y eran recuperables, tal como lo muestran los Doctores Hasbun y Muñoz en el caso presentado ${ }^{10}$.
Gracias a la implementación de medidas de salud pública como la mayor cobertura de atención primaria, atención profesional del parto y la introducción de la vacuna, se ha registrado una disminución sostenida del tétanos a nivel mundial. En Chile, el Programa Ampliado de Inmunizaciones se estableció en el período 1978-1979, cuatro años antes ya se había implementado la vacuna obligatoria, segura y gratuita para la protección de enfermedades transmisibles, como el tétanos, difteria y tos convulsiva ${ }^{11}$. A partir de entonces, la incidencia y mortalidad por la enfermedad mostraron un franco descenso, siendo rarísimo en la actualidad. No obstante, no es infrecuente encontrar reportes de casos aislados en la literatura médica más reciente, especialmente en pacientes no inmunizados o con inmunización parcial ${ }^{12,13}$.

En estos años en que corremos riesgo de caídas en las tasas de vacunación debido a la proliferación de los movimientos anti-vacunas, sumado a la postergación de actividades de supervisión de salud, incluyendo la inmunización, producto de la pandemia de COVID-19 $9^{14,15}$, es posible que volvamos a enfrentarnos a entidades nosológicas que parecían erradicadas ${ }^{16}$. Por este motivo, parece de gran relevancia recordar las manifestaciones clínicas del tétanos y de otras temibles enfermedades, que forman parte de la historia de la medicina.

\section{Conflicto de intereses}

Los autores declaran no tener conflicto de intereses.

\section{Referencias}

1. Vicent $\mathrm{P}$, Venturino H. Antecedentes epidemiológicos del tétanos en chile, 1970-1975. Boletín de la Oficina Sanitaria Panamericana. 1976. [Accedido el 20 de mayo de 2021] Disponible en https://iris.paho.org/bitstream/ handle/10665.2/17526/v81n5p414. pdf? sequence $=1$ \&isAllowed $=y$.

2. Ledermann W. La alferecía y los primeros casos de tétanos neonatal descritos en Chile en 1894. Rev Chil Infectol. 2011;28(6):599-602.

3. Garcés H, Muñoz R. Tetanos Neonatorum. Rev Chil Pediatr. 1955;26(4):141-50.

4. Manterola A, Meneghello J. Tétanos del recién nacido: Consideraciones clínicas sobre 25 casos. Rev Chil Pediatr. 1949;20(9):365-70.

5. Arriagada $\mathrm{P}$, Pineda O. Tratamiento del tétano en el niño. Rev Chil Pediatr. 1948;19(1-12):418-27.
6. Valenzuela MT. Desarrollo y futuro del Programa Ampliado de Inmunizaciones en Chile. Rev Chil Infectol. 2001;18(Suppl 1): 31-6.

7. Aldunate G, Vignau A, Barttlet L, Silva M. Tétanos en el niño. Rev Chil Pediatr. 1965(1):9-15.

8. Cueto L. Ese antiguo Hospital Arriarán... y su hermoso parque. Boletin Servicio de Pediatria Hospital Clinico San Borja Arriarán. Departamento de Pediatria Universidad de Chile 2010;9(42):10-1.

9. Cordero J, Olivos P, Galled A, et al. Tetanos. Rev Chil Pediatr. 1977;48(1):510.

10. Davis PR, Rowland HAK. Vertebral fractures in west Africans suffering from tetanus: a clinical and osteological study. J Bone Joint Surg Br. 1965;47(1):61-71.

11. González C. Programa nacional de inmunización en Chile, pasado, presente y futuro. Rev Med Clín Las Condes 2020;31(3):225-32.
12. Cejudo-García de Alba MDP, Valle-Leal JG, Sánchez JG, Vázquez-Amparano ADJF. Tétanos, una enfermedad vigente en población pediátrica: Reporte de un caso. Rev Chil Pediatr. 2017;88(4):507-10.

13. Armijo MJ, Soto-Aguilar BF, Brito AC. Tétanos generalizado: caso clínico y revisión del tema. Rev Chil NeuroPsiquiatr. 2012;50(4):229-33.

14. Santoli JM, Lindley MC, DeSilva MB, et al. Effects of the COVID-19 Pandemic on Routine Pediatric Vaccine Ordering and Administration-United States, 2020. MMWR Morb Mortal Wkly Rep 2020;69:591-3.

15. Bastias M, Brstilo I, González C. Vacunación programática 2020 en Chile en tiempos de pandemia de SARS-CoV-2. Rev Chil Infectol. 2021:38(3). En prensa.

16. Pan American Health Organization/ World Health Organization. Epidemiological Update: Diphtheria. 17 November 2020, Washington, D.C.: $\mathrm{PAHO} / \mathrm{WHO} ; 2020$. 